\title{
Analysis of bacterial phospholipid markers and plant monosaccharides during forage degradation by Ruminococcus flavefaciens and Fibrobacter succinogenes in co-culture
}

\author{
Liliana Saluzzi, ${ }^{1}$ Alistair Smith ${ }^{2}$ and Colin S. Stewart ${ }^{1 *}$ \\ ${ }^{1}$ Rowett Research Institute, Bucksburn, Aberdeen AB2 9SB, UK \\ ${ }^{2}$ The Macaulay Land Use Research Institute, Cragiebuckler, Aberdeen AB9 2QJ, UK
}

(Received 25 March 1993; revised 25 May 1993; accepted 7 June 1993)

\begin{abstract}
Marker components of the phospholipids of Ruminococcus flavefaciens and Fibrobacter succinogenes were identified for studies on the degradation of forage by these bacteria growing in mixed culture. The principal fatty acid methyl esters and dimethyl acetals detected varied between strains and were influenced by the addition of a mixture of higher volatile fatty acids and vitamins to the medium, but these effects were small compared to the differences between the species. When two strains of $R$. flavefaciens were grown on a mixture of clover and ryegrass, and on barley straw in the presence or absence of two strains of $F$. succinogenes, the solubilization of plant material tended to be lowered by the presence of $F$. succinogenes. $R$. flavefaciens was the predominant bacterium among colonies recovered from roll tubes, and the phospholipids were primarily those of $R$. flavefaciens. Analysis of the culture supernatant liquids showed that $F$. succinogenes produced greater amounts of free and bound xylose from both clover and straw than did $R$. flavefaciens. With both forages, cultures containing the two species produced more soluble free arabinose, and less soluble-bound arabinose, than either species grown alone.
\end{abstract}

\section{Introduction}

Interactions between cellulolytic micro-organisms from the rumen and from other habitats have mainly been monitored by comparing the cellulolytic or plant cellwall-degrading activity of pure and mixed cultures in which the species are represented by single selected strains (Dehority \& Scott, 1967; Stewart \& Walsh, 1972; Miron, 1991; Irvine \& Stewart, 1991; Bernalier et al., 1992). Assessing the contribution of different bacteria to the activity of mixed cultures would be helped if the relative contributions of the component species to the microbial biomass were known.

The analysis of microbial lipids has been used for the estimation of the relative proportions of microbial species and groups in natural mixed microbial communities (Bobbie \& White, 1980; McKinley \& Vestal, 1984; Vestal \& White, 1989). Although the composition of the lipids, including phospholipids and plasmalogens, of many rumen bacteria has been determined (Verkely $e t$ al., 1975; Miyagawa, 1982; Watanabe et al., 1982), little

* Author for correspondence. Tel. +44 224716 654; fax +44 224 715349 .

Abbreviations: DMA, dimethyl acetal; FAME, fatty acid methyl ester; i, iso; ai, anteiso. attempt has so far been made to use lipid markers for the analysis of mixed cultures of rumen organisms.

The main aim of this study was to identify potential markers among the alkyl and alk-1-enyl components of the phospholipids of strains of $F$. succinogenes and $R$. flavefaciens, and to study the effect of the presence of $F$. succinogenes on the fermentation of fibrous substrates by $R$. flavefaciens. To reduce possible bias resulting from strain selection, and for a closer approximation to natural conditions, two strains of the relevant species (the type strain and one other) were used simultaneously in the present study. The growth substrates, a mixture of white clover and ryegrass harvested from a mixed sward, and a pure sample of barley straw, were selected as representative of mixed legume/grass pasture forage and cereal straw respectively, as such plants are known to differ in their chemical composition and susceptibility to attack by rumen bacteria (Varel et al., 1989).

\section{Methods}

Bacteria. The type strains, FD1 of Ruminococcus flavefaciens and S85 of Fibrobacter succinogenes were obtained from M. P. Bryant, University of Illinois, Urbana, IL, USA. The isolation and characteristics of $F$. succinogenes strain BL2 was as described by Stewart et al. (1981) and $R$. flavefaciens strain 17 was isolated and characterized by Flint $e t$ al. (1989). 
Anaerobic methods and viable counts. Media were prepared and maintained under anaerobic conditions using $\mathrm{O}_{2}$-free $\mathrm{CO}_{2}$ as described by Bryant (1972). The bacteria were maintained using medium M3, a modification of medium M2 of Hobson (1969) from which lactate was omitted, contained in $16 \times 125 \mathrm{~mm}$ glass Hungate tubes sealed with butyl rubber septum stoppers (Bellco Glass). For some tests referred to in the text, the medium was supplemented with vitamins and volatile fatty acids. The vitamin solution contained (per $100 \mathrm{ml}$ distilled $\mathrm{H}_{2} \mathrm{O}$ ): $15 \mathrm{mg}$ pyridoxamine, $5 \mathrm{mg}$ (each) folic acid, riboflavin and thiamine, $3 \mathrm{mg} p$-aminobenzoic acid (PABA), and $1 \mathrm{mg}$ (each) biotin and cobalamin. The VFA solution contained (per $100 \mathrm{ml}$ distilled $\mathrm{H}_{2} \mathrm{O}$ ) $4 \mathrm{ml} n$-butyric acid and $1 \mathrm{ml}$ (each) $n$-valeric, iso-valeric, iso-butyric and DL-2-methylbutyric acids. The $\mathrm{pH}$ of this solution was adjusted to 7.5 with $\mathrm{NaOH}$. Viable counts were performed (triplicate) in roll tubes of medium M3, by the method of Hungate (1969). Counts of bacteria detached from clover or straw were carried out following detachment with methylcellulose at a final concentration of $0.15 \%(\mathrm{w} / \mathrm{v})$ (Product 29779 , high degree of substitution, Merck) and chilling, as described by Minato \& Suto (1981). The proportions of $R$. flavefaciens and $F$. succinogenes present in the mixed cultures was determined by the examination of Gram-stained smears of (on average) 25 colonies picked from roll tubes which received the highest countable dilutions of samples from each experimental flask. Strains 17 and FD1 of $R$. flavefaciens were distinguished on the basis of the difference in the average number of cells in the chains, in the intensity of their Gramstaining reaction, differences in their ability to solubilize cotton fibres (Stewart et al., 1981) and differences in the composition of their phospholipids, as described in the text. All culture incubations were at $38 \pm 1^{\circ} \mathrm{C}$.

Growth of bacteria on clover and barley straw. Winter barley straw (Hordeum vulgare) was the untreated straw used by Kolankaya $e t$ al. (1985). The mixture of white clover (Trifolium repens) var. Kersey (62\% of dry matter) and ryegrass (38\% of dry matter) was from a mixed sward harvested at the Scottish Agricultural College, Aberdeen. The forages were hammer-milled and fractionated by dry-sieving (Endecotts Filters) to a particle size of 212 to $425 \mu \mathrm{m}$. To reduce potential interference from plant lipids, lipids were extracted from the forages before incubation with bacteria. Extraction was performed (twice) by boiling the forages under reflux with chloroform:methanol $\left[2: 1, \mathrm{v} / \mathrm{v} ; 30 \mathrm{ml}(\mathrm{g} \mathrm{sample})^{-1}\right]$ for $1 \mathrm{~h}$ as recommended by Christie (1982). After extraction, residual solvent was removed by air drying. Plant material $(3.0 \mathrm{~g})$ was added to $1000 \mathrm{ml}$ capacity Duran Bottles (Gallenkamp) which received $600 \mathrm{ml}$ medium M3 from which sugars were omitted. The bottles were fitted with ports for inoculation constructed from Hungate tubes (above) from which the sealed end had been cut. The inocula were pre-grown for $18-24 \mathrm{~h}$ on medium M3 modified to contain cellobiose $(0 \cdot 25 \% \mathrm{w} / \mathrm{v})$ as sole energy source. The inoculum volume was $5 \%$ of the culture volume. The results reported here are the pooled observations from three separate experiments. To standardize the inoculum between experiments as far as possible, the relationship between $\mathrm{OD}_{650}$ and culture counts was determined for all four strains growing on medium $M 3$, and the $\mathrm{OD}_{650}$ of the inoculum was adjusted to 0.35 for $R$. flavefaciens and 0.9 for $F$. succinogenes.

Isolation and analysis of bacterial phospholipids. Bacteria from liquid supernatants or detached from plant material with methylcellulose were washed twice in distilled $\mathrm{H}_{2} \mathrm{O}$, recovered by centrifugation $\left(20000 \mathrm{~g}, 20 \mathrm{~min}\right.$ ) and resuspended in a known volume of distilled $\mathrm{H}_{2} \mathrm{O}$. Lipids were extracted by the method of Folch et al. (1957) with some modifications. Methanol was added to a final ratio methanol: $\mathrm{H}_{2} \mathrm{O}$ of 1.33:1 (v/v). The pellets were homogenized for $1 \mathrm{~min}$ using an IKA Ultra-Turrax T25 homogenizer (Janke \& Kunkel). A volume of chloroform, double that of the methanol, was added and the homogenization repeated for $2 \mathrm{~min}$. The phases were separated by centrifugation. The interfacial material and the glass sample container were washed twice with chloroform and, together with the lower phase containing the extracted lipids, the washings were filtered through a bed of anhydrous $\mathrm{Na}_{2} \mathrm{SO}_{4}$ on Whatman IPS filter paper which had been soaked previously with chloroform. The solvent was removed in a rotary film evaporator at $40^{\circ} \mathrm{C}$ and the total lipid fraction was dried azeotropically three times under nitrogen after adding methanol to remove traces of $\mathrm{H}_{2} \mathrm{O}$. Lipids from bacteria attached to plant material were extracted by the same method except that after the first extraction with solvent, the extract was filtered through Whatman no. 1 filter paper and the solid residue resuspended in chloroform:methanol (2:1, $\mathrm{v} / \mathrm{v})$. The extraction was repeated. After filtering and washing the residues, the extracts were pooled, $\mathrm{H}_{2} \mathrm{O}$ added and the procedure continued as described above.

Lipid classes were separated by adsorption column chromatography using Si Silica Bond Elut columns (Analytichem International). After conditioning the columns with methanol and chloroform, neutral lipids were removed under vacuum with three column volumes of chloroform, glycolipids with chloroform: acetone $(1: 1, \mathrm{v} / \mathrm{v})$, and phospholipids and plasmalogens with methanol. Thin layer chromatography (Whatman Silica Gel KS 150 A plates, $250 \mu \mathrm{m}$ ) was used to verify the purity of the lipid fractions obtained (Christie, 1982). The plates (normally $20 \times$ $20 \mathrm{~cm}$ ) were developed with a mixture of chloroform:methanol: $\mathrm{H}_{2} \mathrm{O}$ $(65: 25: 4$, by vol.). After drying the plates in air, further separation of free fatty acids and neutral lipids from phospholipids was obtained whilst developing the plates with a solution of $10 \%(\mathrm{v} / \mathrm{v})$ diethyl ether in petroleum ether plus formic acid [10 drops $\left.(100 \mathrm{ml})^{-1}\right]$. Glycolipids were detected by developing the plates using acetone:acetic acid: $\mathrm{H}_{2} \mathrm{O}$ (100:2:1, by vol.). Fatty acid methyl esters (FAMEs) and dimethyl acetals (DMAs) were prepared by acid-catalysed transesterification of phospholipids. The pure phospholipid fraction, dissolved in tetrahydrofuran, was heated at $100^{\circ} \mathrm{C}$ in sealed tubes under $\mathrm{N}_{2}$ with $5 \%$ methanolic $\mathrm{HCl}$ [10 ml (mg lipid) $\left.{ }^{-1}\right]$ for $1.5 \mathrm{~h}$ (Christie, 1982). Water was added to the cooled samples and the derivatives were extracted with hexane, followed by diethyl ether. The extracts were pooled, washed twice with distilled $\mathrm{H}_{2} \mathrm{O}$ and dried with anhydrous $\mathrm{Na}_{2} \mathrm{SO}_{4}$. FAMEs and DMAs were analysed by capillary GC, using a WCOT fused silica column $(50 \mathrm{~m}$ length, $0.25 \mathrm{~mm}$ i.d.) coated with a polar liquid phase CP-sil-88 (film thickness $0.21 \mu \mathrm{m}$ : Chrompack). Injector and flame ionization detectors were at $280^{\circ} \mathrm{C}$. Helium $\left(0.5 \mathrm{ml} \mathrm{min}{ }^{-1}\right)$ was the carrier gas. The column was operated at $150^{\circ} \mathrm{C}$ for $4 \mathrm{~min}$ followed by a $4{ }^{\circ} \mathrm{C} \mathrm{min}{ }^{-1}$ rise to $220^{\circ} \mathrm{C}$. Injection was performed in split mode. The relative retention time (RRT) of the peaks was compared with that of 2-methylhexadecanoate, synthesized at the Rowett Institute. Peaks were identified by comparison of the RRT with those of standards (Sigma; Supelchem). Peak areas of the chromatographic components determined by disk integration were calculated using an HP 3390A integrator (Hewlett Packard). Further identification of components for which standards were not commercially available was by GC-MS.

Numerical analysis of phospholipid profiles. Similarities were calculated with the Bravais-Pearson coefficient of linear correlation (Drucker, 1976). The square roots of the data were used to reduce the influence of the larger peaks on the analysis.

Chemical analysis of plant residues. The residues remaining after fermentation were recovered by filtration (Whatman no. 1 filter paper), washed twice with distilled $\mathrm{H}_{2} \mathrm{O}$, and lyophilized before weighing. Samples were hydrolysed in $12 \mathrm{M}-\mathrm{H}_{2} \mathrm{SO}_{4}$ for $30 \mathrm{~min}$ at $25^{\circ} \mathrm{C}$ with regular vortex mixing, then at $100^{\circ} \mathrm{C}$ for $2 \mathrm{~h}$ after dilution to $1 \mathrm{M}$ $\mathrm{H}_{2} \mathrm{SO}_{4}$ (Hoebler et al., 1989). Aliquots of the hydrolysates were neutralized, reduced with sodium borohydride in dilute ammonia solution, extracted and analysed by GC using $\beta$-D-allose as internal standard (Englyst \& Cummings, 1984).

Sugars in culture supernatants were analysed in the same way following clarification with zinc acetate in acetic acid (Carrez I) and 
Table 1. Phospholipid DMA and FAME profiles of strains $S 85$ and BL2 of F. succinogenes and strains 17 and FD1 of $R$. flavefaciens grown in medium M3 with and without the addition of volatile fatty acids and vitamins $(M 3+V V)$

\begin{tabular}{|c|c|c|c|c|c|c|c|c|}
\hline \multirow{3}{*}{$\begin{array}{l}\text { Strain: } \\
\text { Medium: }\end{array}$} & \multicolumn{4}{|c|}{ F. succinogenes } & \multicolumn{4}{|c|}{ R. flavefaciens } \\
\hline & \multicolumn{2}{|c|}{ S85 } & \multicolumn{2}{|c|}{ BL2 } & \multicolumn{2}{|r|}{17} & \multicolumn{2}{|c|}{ FD1 } \\
\hline & M3 & $\mathrm{M} 3+\mathrm{VV}$ & M3 & $\mathrm{M} 3+\mathrm{VV}$ & M3 & $\mathrm{M} 3+\mathrm{VV}$ & M3 & $\mathrm{M} 3+\mathrm{VV}$ \\
\hline \multicolumn{9}{|l|}{ DMA } \\
\hline $11: 0$ & 0.3 & 0.2 & - & - & - & - & - & - \\
\hline $12: 0$ & $5 \cdot 7$ & $2 \cdot 8$ & $1 \cdot 6$ & 0.9 & - & - & - & - \\
\hline $13: 0$ & 3.9 & $3 \cdot 3$ & $6 \cdot 4$ & $5 \cdot 6$ & - & - & - & - \\
\hline $14: 0$ & 6.9 & 3.9 & 3.9 & 1.8 & 0.4 & 0.4 & 0.5 & 0.4 \\
\hline $15: 0$ & 0.7 & 0.9 & 0.3 & 0.2 & 0.8 & 0.7 & $1 \cdot 3$ & 1.0 \\
\hline $16: 0$ & 0.5 & 0.5 & - & - & $11 \cdot 1$ & $9 \cdot 3$ & $12 \cdot 2$ & $9 \cdot 8$ \\
\hline $17: 0$ & - & - & - & - & 0.6 & 0.3 & 0.3 & 1.0 \\
\hline 18:0 & - & - & - & - & $5 \cdot 1$ & $4 \cdot 4$ & 0.6 & 0.9 \\
\hline ai13:0 & $11 \cdot 2$ & $9 \cdot 1$ & $7 \cdot 3$ & $10 \cdot 1$ & $0 \cdot 3$ & $0 \cdot 3$ & 0.4 & 0.2 \\
\hline ai15:0 & 1.7 & 1.6 & $1 \cdot 4$ & $1 \cdot 3$ & 0.4 & 0.3 & 0.7 & 1.0 \\
\hline ai17:0 & - & - & - & - & 1.6 & $1 \cdot 3$ & $2 \cdot 5$ & $3 \cdot 3$ \\
\hline i12:0 & $1 \cdot 0$ & 0.7 & 0.6 & 0.4 & - & - & - & - \\
\hline i13:0 & 0.1 & 0.2 & - & - & - & - & - & - \\
\hline i14:0 & $3 \cdot 5$ & $4 \cdot 5$ & $5 \cdot 1$ & $3 \cdot 3$ & 0.1 & $0 \cdot 1$ & 0.3 & - \\
\hline i15:0 & - & - & - & - & $2 \cdot 5$ & $5 \cdot 1$ & $8 \cdot 7$ & $15 \cdot 8$ \\
\hline i17:0 & 0.2 & $0 \cdot 1$ & - & - & $10 \cdot 9$ & $14 \cdot 1$ & $6 \cdot 1$ & $8 \cdot 4$ \\
\hline \multicolumn{9}{|l|}{ FAME } \\
\hline $13: 0$ & 1.4 & 1.0 & $2 \cdot 1$ & 3.6 & 0.1 & - & 0.1 & - \\
\hline $14: 0$ & $13 \cdot 2$ & 9.9 & $16 \cdot 8$ & $9 \cdot 3$ & 1.0 & 0.7 & 1.2 & 0.9 \\
\hline $15: 0$ & $11 \cdot 1$ & $12 \cdot 1$ & $22 \cdot 0$ & 29.8 & $1 \cdot 3$ & 0.7 & 1.6 & 0.9 \\
\hline $16: 0$ & $20 \cdot 0$ & $23 \cdot 6$ & $12 \cdot 8$ & $9 \cdot 1$ & 9.8 & $6 \cdot 1$ & $12 \cdot 3$ & $8 \cdot 3$ \\
\hline $17: 0$ & 3.9 & 4.8 & $1 \cdot 4$ & 1.9 & 0.3 & $0 \cdot 1$ & 0.3 & - \\
\hline $18: 0$ & $2 \cdot 8$ & $2 \cdot 4$ & 0.7 & 0.5 & 1.8 & 1.2 & $1 \cdot 2$ & 1.0 \\
\hline ai15:0 & 5.4 & $8 \cdot 1$ & 6.9 & $13 \cdot 6$ & $3 \cdot 0$ & 1.9 & $4 \cdot 7$ & $4 \cdot 3$ \\
\hline ai17:0 & $2 \cdot 0$ & 2.9 & 0.8 & 1.4 & $4 \cdot 5$ & $2 \cdot 2$ & 3.0 & $2 \cdot 3$ \\
\hline i14:0 & $2 \cdot 1$ & $2 \cdot 1$ & 5.9 & 4.5 & 0.1 & 0.2 & 0.3 & 0.2 \\
\hline i15:0 & - & 0.7 & - & - & $29 \cdot 3$ & 38.9 & 37.0 & $36 \cdot 4$ \\
\hline i16:0 & 1.3 & $3 \cdot 2$ & 3.9 & $2 \cdot 5$ & $4 \cdot 2$ & 1.8 & 1.5 & 1.4 \\
\hline i17:0 & 0.3 & 0.7 & - & 0.2 & 10.5 & 8.9 & 3.0 & $2 \cdot 6$ \\
\hline i18:0 & 0.4 & 0.3 & - & - & 0.3 & 0.3 & - & - \\
\hline
\end{tabular}

potassium ferricyanide (Carrez II) solutions (Feeding Stuffs Regulations, 1982) to remove protein and other sources of interference.

\section{Results}

\section{Phospholipid content and identification of marker} components

Analysis of freeze-dried cells of strains of $R$. flavefaciens (Gram-positive) and F. succinogenes (Gram-negative) revealed that the total phospholipid content (percentage of dry matter) of $F$. succinogenes $(5 \cdot 4 \pm 0.4, n=8)$ was more than twice that of $R$. flavefaciens $(2 \cdot 1 \pm 0 \cdot 3, n=8)$. To determine the possible effect of either cross-feeding of or competition for trace nutrients in the mixed cultures, the phospholipid content of the strains was determined in the presence and absence of vitamin and VFA supplements in the medium. Neither the strain identity nor these additions to the medium markedly affected the phospholipid content of the cells (data not shown).

When grown on medium M3 with or without the addition of vitamins and VFA, there were a number of marked differences between the species in the relative proportions of the individual FAMEs and DMAs detected, and in both species, some variation between strains (Table 1). In general, the two strains of $R$. flavefaciens contained a higher proportion of DMAs $16: 0, \mathrm{i} 15: 0$ and $\mathrm{i} 17: 0$, and a lower proportion of DMAs ai13:0, 13:0, i14:0 and 14:0 than $F$. succinogenes. The $R$. flavefaciens strains contained more FAMEs i15:0 and i17:0, and a lower proportion of FAMEs 14:0 and 15:0 than $F$. succinogenes. The addition of vitamins and VFA to the culture medium produced changes in the relative proportions of some of the DMA and FAME components of the two species, but these changes were 
Table 2. Correlation between the phospholipid profiles of $R$. flavefaciens (strains 17 and FD1) and F. succinogenes (strains S85 and BL2) cells in the inoculum (grown on cellobiose) or recovered following growth on clover or straw for different incubation times

Results are expressed as the coefficient of linear correlation between the average profile of the same species grown on the different substrates.

\begin{tabular}{|c|c|c|c|c|c|c|}
\hline \multirow[b]{2}{*}{$\begin{array}{l}\text { Incubation } \\
\text { time (d) }\end{array}$} & \multicolumn{3}{|c|}{ R. flavefaciens } & \multicolumn{3}{|c|}{ F. succinogenes } \\
\hline & $\begin{array}{l}\text { Clover } \\
\text { vs } \\
\text { straw }\end{array}$ & $\begin{array}{l}\text { Inoculum } \\
\text { vs } \\
\text { clover }\end{array}$ & $\begin{array}{l}\text { Inoculum } \\
\text { vs } \\
\text { straw }\end{array}$ & $\begin{array}{l}\text { Clover } \\
\text { vs } \\
\text { straw }\end{array}$ & $\begin{array}{l}\text { Inoculum } \\
\text { vs } \\
\text { clover }\end{array}$ & $\begin{array}{c}\text { Inoculum } \\
\text { vs } \\
\text { straw }\end{array}$ \\
\hline 2 & 0.99 & 0.92 & 0.95 & 0.99 & 0.95 & 0.93 \\
\hline 3 & 0.99 & 0.92 & 0.95 & 1.00 & 0.92 & 0.91 \\
\hline 7 & 0.99 & 0.96 & 0.98 & 0.98 & 0.94 & 0.91 \\
\hline
\end{tabular}

generally small in relation to the differences between the species. Thus, the addition of vitamins and VFA to the medium led to a decrease in the proportion of DMAs 12:0, 14:0 and 112:0, and FAME 14:0 and an increase in the proportion of FAME ai15:0 in the phospholipids of both strains of $F$. succinogenes. The proportion of FAMEs 15:0 and 16:0 in strain BL2, and of FAMEs 16:0 and 17:0 in strain S85 were also modified by the addition of vitamins and VFA to the medium. The addition of vitamins and VFA increased the proportions of DMAs i15:0 and i17:0 in both strains of $R$. flavefaciens, and influenced the proportion of FAMEs i15:0 and ai17:0 in the phospholipids of strain 17. The data in Table 1 also suggested that the two strains of $R$. flavefaciens could be distinguished on the basis of the differences in the relative proportions of DMAs i15:0 and i17:0, and FAME i17:0 present.

Following analysis of the phospholipid FAMEs and DMAs in the biomass detached from the plant material with methylcellulose, coefficients of correlation were calculated to establish whether the composition of the phospholipids of either $R$. flavefaciens or $F$. succinogenes was markedly affected by either the growth substrate or the duration of incubation. This showed that the composition of the $R$. flavefaciens and $F$. succinogenes populations was relatively little affected by these variables, despite the fact that for each species, two strains were present (Table 2).

The phospholipid markers for calculation of the relative biomass of the two species in mixed cultures were chosen on the basis that the marker occurred in concentrations of less than $1 \%$ in the second species and none of the markers were present at concentrations of more than $1 \%$ of the residual lipids in the plant material and nutrient medium. The DMAs ai13:0, 13:0, i14:0 and 14:0 were selected as markers for $F$. succinogenes, and DMAs i15:0, 16:0, i17:0 and 18:0, and FAME i15:0 were selected for $R$. flavefaciens. Although 18:0

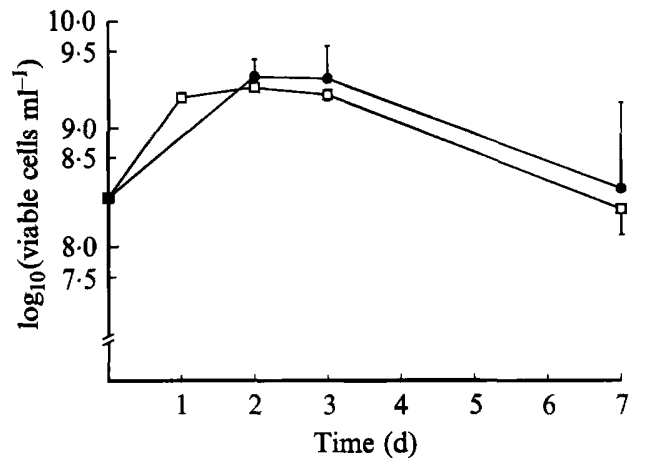

Fig. 1. Total viable cell counts recovered from cultures inoculated with $R$. flavefaciens and $F$. succinogenes and grown on clover/ryegrass ( $\square$ ) or barley straw (O). Bars indicate SD.

DMA was detected in only small amounts in $R$. flavefaciens strain FD1 in the experiment shown in Table 1 , this component was routinely detected in this strain subsequently, constituting around $3.4 \%$ of the DMA.

\section{Mixed cultures - biomass analysis and viable counts}

When $R$. flavefaciens strains 17 and FD1 were grown together in the presence of $F$. succinogenes strains S85 and BL2, the viable counts and examination of colonies picked from the roll tubes suggested that $R$. flavefaciens quickly became predominant. Thus, $F$. succinogenes contributed $59 \%$ of the colonies recovered after $1 \mathrm{~d}$ incubation on clover, and none of the colonies recovered after 2,3 or $7 \mathrm{~d}$ incubation on clover. Of the colonies recovered from cultures grown on straw, $F$. succinogenes contributed $13 \%$ after $2 \mathrm{~d}$ incubation, none after $3 \mathrm{~d}$ incubation, and $20 \%$ after $7 \mathrm{~d}$ incubation. The viable counts from the cultures inoculated with both species, corrected for the number of cells in the chains of the ruminococci, are shown in Fig. 1 . The average $(n=50)$ chain length was $16 \cdot 8 \pm 10 \cdot 6$ cells for strain FD1, and 


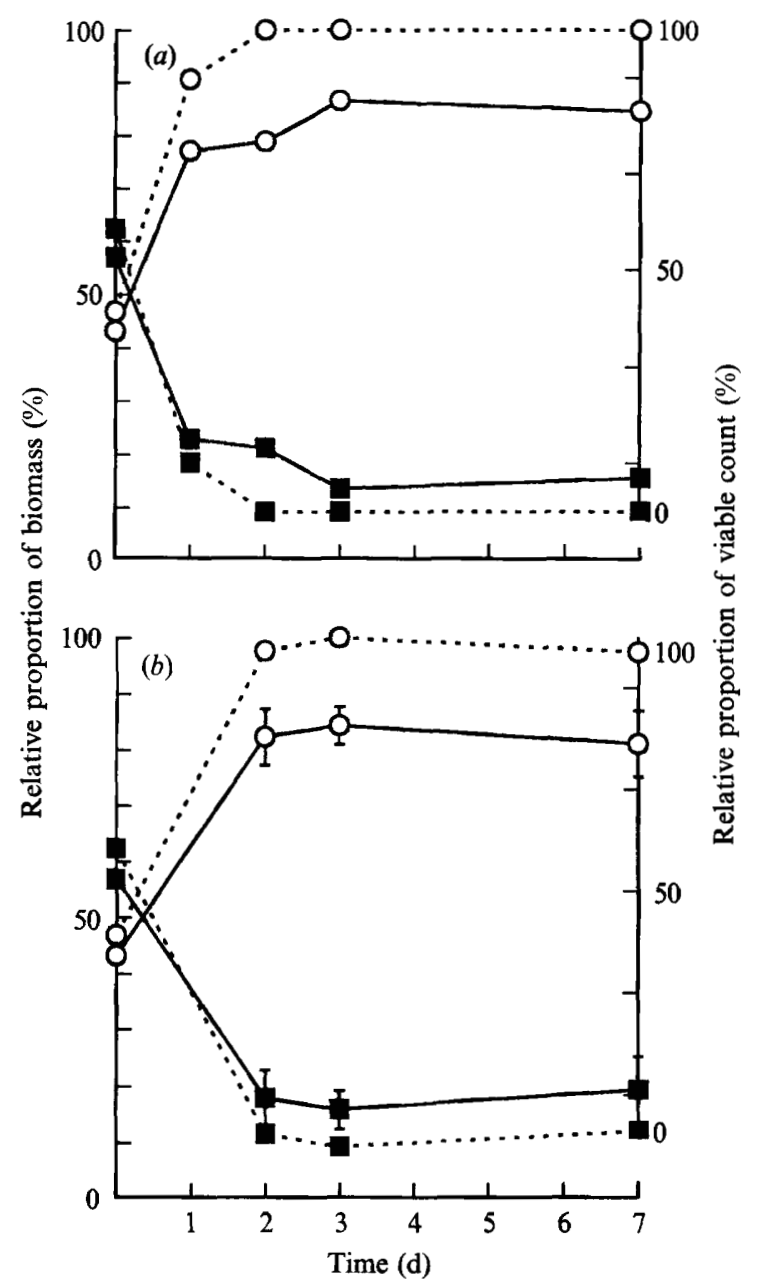

Fig. 2. Relative proportions of $R$. flavefaciens $(O)$ and $F$. succinogenes (D) in mixed culture at different stages of incubation on clover/ryegrass (a) or barley straw $(b)$. Results are presented as the percentage of the total biomass from phospholipid analysis (-), and as the percentage of total viable cells recovered ( ...). Bars indicate SD.

$4 \cdot 8 \pm 2 \cdot 4$ for strain 17 . Particularly when clover was the substrate, cultures inoculated with ruminococci alone and incubated for 2 or $3 \mathrm{~d}$ tended to contain more recoverable viable cells than those shown in Fig. 1 (between $3.6 \times 10^{9}$ and $1.5 \times 10^{10}$ cells ml $^{-1}$ ), though this tendency was not always statistically significant. No significant increase was found in the viable numbers of $F$. succinogenes recovered from either clover or straw during the incubations, the viable counts ranging from $5 \times 10^{7}$ to $5 \times 10^{8} \mathrm{ml}^{-1}$ during the first $3 \mathrm{~d}$ incubation with either substrate, then falling to between $2 \times 10^{7}$ and $4 \times 10^{7} \mathrm{ml}^{-1}$ at $7 \mathrm{~d}$. However, this species degraded both clover and straw (Fig. 3 below). When Gram-stained smears of the ruminococci picked from the highest countable dilutions were examined, the majority of the colonies $(75 \%$ for clover and $56 \%$ for straw) were found to belong to strain 17. No attempt was made to distinguish between the two strains of $F$. succinogenes: neither strain formed chains

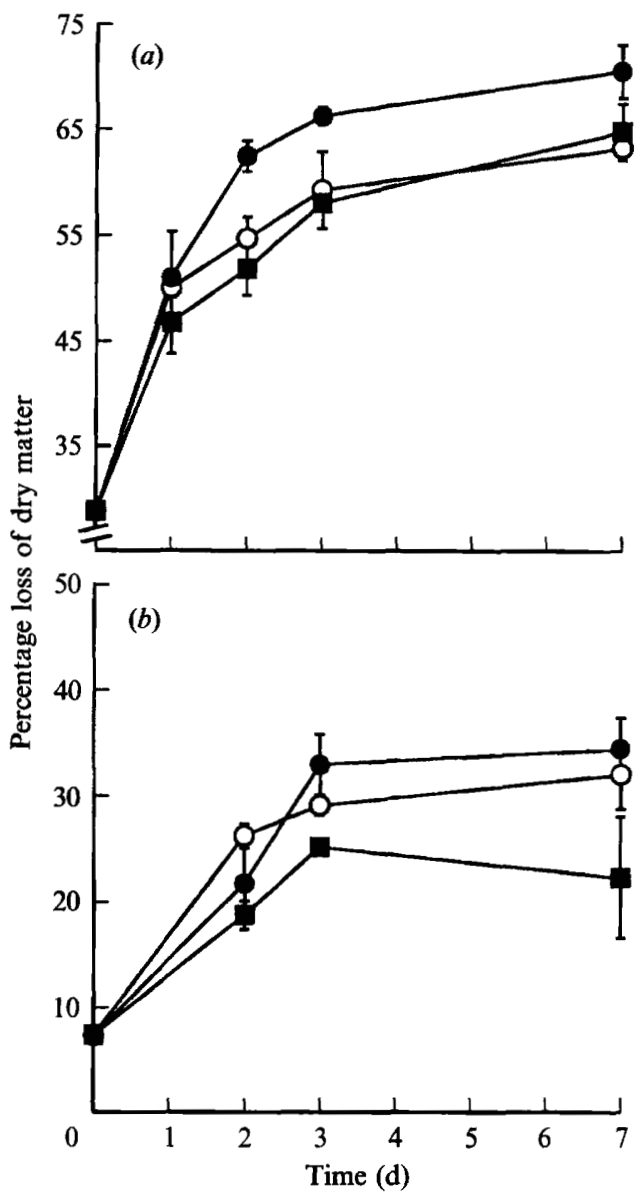

Fig. 3. Solubilization of dry matter (\%) of clover/ryegrass (a) and barley straw $(b)$ by $R$. flavefaciens $(\boldsymbol{O}), F$. succinogenes $(\boldsymbol{D})$ and the coculture containing both species $(O)$ at different stages of incubation. Bars indicate SD.

or clumps and they could not readily be differentiated by microscopic examination.

The analysis of the phospholipids in the bacterial population detached from the plant material using methylcellulose suggested that with both clover and straw, $R$. flavefaciens became prodominant in the early stages of the incubations despite forming a smaller proportion of the biomass in the inoculum than $F$. succinogenes, the attempts to standardize the inoculum having failed to ensure precisely matching numbers of the two species (Fig. $2 a, b$ ). The relative proportions of each species in the cultures containing both $R$. flavefaciens and $F$. succinogenes shown in Fig. 2 were calculated by comparing the fatty acid profiles with those of the cultures containing single species incubated with the same forage for the same time. The estimates of the relative viable numbers of the two species in mixed culture (dotted lines in Fig. $2 a, b$ ) followed a similar pattern to the relative proportions of the bacteria in the biomass calculated from analysis of phospholipids. 
Table 3. Fate of monosaccharides of clover/ryegrass and barley straw following incubations with $R$. flavefaciens strains 17 and FD1, Fibrobacter succinogenes strains $S 85$ and BL2, and cultures containing all four strains

Results are the means of two replicates. The average coefficient of variation was 0.16 .

\begin{tabular}{|c|c|c|c|c|}
\hline \multirow[b]{2}{*}{ Fraction } & \multirow{2}{*}{$\begin{array}{c}\text { Starting } \\
\text { amount } \\
\text { (mg) }\end{array}$} & \multicolumn{3}{|c|}{$\begin{array}{l}\text { Amount remaining after incubation for } 7 \mathrm{~d} \\
\qquad(\mathrm{mg})\end{array}$} \\
\hline & & $\begin{array}{c}R . \\
\text { flavefaciens }\end{array}$ & $\begin{array}{c}F . \\
\text { succinogenes }\end{array}$ & Co-culture \\
\hline \multicolumn{5}{|l|}{ Clover } \\
\hline \multicolumn{5}{|l|}{ Arabinose } \\
\hline insoluble & $86 \cdot 9$ & $14 \cdot 4$ & $21 \cdot 1$ & 14.8 \\
\hline soluble monosacc. & $8 \cdot 6$ & 33.9 & $25 \cdot 6$ & $57 \cdot 6$ \\
\hline soluble-bound & 30.6 & $79 \cdot 2$ & 91.5 & $54 \cdot 6$ \\
\hline Total & $126 \cdot 1$ & $127 \cdot 5$ & $138 \cdot 2$ & 127.0 \\
\hline \multicolumn{5}{|l|}{ Xylose } \\
\hline insoluble & $233 \cdot 7$ & $43 \cdot 4$ & 96.7 & $60 \cdot 6$ \\
\hline soluble monosacc. & $19 \cdot 3$ & $1 \cdot 1$ & $9 \cdot 4$ & 0.7 \\
\hline soluble-bound & 0.0 & $50 \cdot 4$ & $109 \cdot 1$ & $63 \cdot 8$ \\
\hline Total & $253 \cdot 0$ & $94 \cdot 8$ & $215 \cdot 2$ & $125 \cdot 1$ \\
\hline \multicolumn{5}{|l|}{ Glucose } \\
\hline insoluble & $740 \cdot 0$ & $100 \cdot 5$ & $199 \cdot 3$ & $141 \cdot 4$ \\
\hline soluble monosacc. & $37 \cdot 7$ & 0.4 & $1 \cdot 1$ & 1.4 \\
\hline soluble-bound & 382.2 & $234 \cdot 6$ & $264 \cdot 6$ & $152 \cdot 7$ \\
\hline Total & 1159.9 & $335 \cdot 5$ & 465.0 & $295 \cdot 5$ \\
\hline \multicolumn{5}{|l|}{ Straw } \\
\hline \multicolumn{5}{|l|}{ Arabinose } \\
\hline insoluble & $132 \cdot 2$ & $47 \cdot 8$ & 87.5 & 88.8 \\
\hline soluble monosacc. & 5.9 & 34.9 & $22 \cdot 7$ & $52 \cdot 9$ \\
\hline soluble-bound & $44 \cdot 9$ & $36 \cdot 0$ & $32 \cdot 8$ & $18 \cdot 7$ \\
\hline Total & 183.0 & $118 \cdot 7$ & $143 \cdot 0$ & $160 \cdot 4$ \\
\hline \multicolumn{5}{|l|}{ Xylose } \\
\hline insoluble & 929.5 & $417 \cdot 1$ & 638.8 & 583.9 \\
\hline soluble monosacc. & $16 \cdot 1$ & 1.8 & 23.4 & 1.8 \\
\hline soluble-bound & 0.0 & $76 \cdot 3$ & 203.8 & $70 \cdot 2$ \\
\hline Total & $942 \cdot 6$ & $495 \cdot 2$ & 866.0 & $655 \cdot 9$ \\
\hline \multicolumn{5}{|l|}{ Glucose } \\
\hline insoluble & $1505 \cdot 0$ & 899.5 & $1224 \cdot 2$ & $1096 \cdot 4$ \\
\hline soluble monosacc. & $40 \cdot 9$ & $1 \cdot 1$ & 0.7 & 1.5 \\
\hline soluble-bound & $403 \cdot 3$ & $143 \cdot 7$ & $94 \cdot 7$ & $84 \cdot 4$ \\
\hline Total & $1949 \cdot 2$ & $1044 \cdot 3$ & $1319 \cdot 6$ & $1182 \cdot 3$ \\
\hline
\end{tabular}

To check whether the process of detaching cells with methylcellulose favoured the detection of one or other of the species, a few analyses were performed of bacterial phospholipids associated with the residual plant material after $3 \mathrm{~d}$ incubation following its treatment with methylcellulose. Analysis of these samples (data not shown) suggested that the population which remained adherent during this treatment also consisted predominantly of $R$. flavefaciens, in that for both clover and straw, the phospholipids extracted from the plant residues were very largely (97-98\%) those of $R$. flavefaciens.

\section{Analysis of plant material}

After incubation with plant material for up to $7 \mathrm{~d}$, it was clear that the $R$. flavefaciens strains were more active than the strains of $F$. succinogenes in solubilizing clover
(Fig. $3 a, b$ ). When straw was the substrate, the effect of the presence of $F$. succinogenes varied according to the incubation time, but from $3 \mathrm{~d}$ onwards tended to depress solubilization (Fig. $3 b$ ). The negative effect of the presence of $F$. succinogenes was also evident from the analysis of the insoluble plant residues. $R$. flavefaciens removed more of the major monosaccharides, glucose, arabinose and xylose from both forages than did $F$. succinogenes or the cultures containing both species (Table 3). However, the fate of soluble monosaccharides, and of soluble compounds from which monosaccharides could be released by acid hydrolysis (di- or oligosaccharides, or glycosides, shown as 'soluble-bound' in Table 3) showed some contrasting trends. Glucose was largely removed from the monosaccharide pool in all of the cultures. When grown on clover, the mixed culture containing both bacterial species tended to be more 
effective in removing glucose from the soluble-bound pool than either of the pure cultures. Arabinose accumulated in the monosaccharide pool of all cultures, but for both forages this accumulation was greatest with the cultures inoculated with the two bacterial species. Although $F$. succinogenes removed rather less xylose from clover and straw than did $R$. flavefaciens, a high proportion of the xylose solubilized by $F$. succinogenes appeared in the soluble-bound pool (Table 3).

To check whether the negative effect of the presence of $F$. succinogenes on the solubilization of forage by $R$. flavefaciens could be due to competition for growth factors, lipid-extracted clover/ryegrass and barley straw were incubated for $7 \mathrm{~d}$ with the two strains of $R$. flavefaciens in the presence and absence of the two strains of $F$. succinogenes, with and without supplementation of the medium with vitamins and VFA as described in Methods. These tests confirmed that the addition of vitamins and VFA did not influence the degradation of either forage, or reduce the negative effect of the presence of $F$. succinogenes (data not shown).

\section{Discussion}

The composition of the phospholipids of $F$. succinogenes and $R$. flavefaciens was consistent with the data from the more limited chemical analysis of these species reported by Miyagawa et al. (1979) and Minato et al. (1990). Although the phospholipid composition of the two species studied here varied between strains and was affected by the composition of the nutrient medium and by growth on different substrates, these effects were small compared with the differences between the species. The selected FAME and DMA marker components allowed the detection of the two species in mixed culture, and showed the same general trends as the viable counts (Fig. 2), despite the fact that phospholipid analysis detects both viable and non-viable cells. Analysis of phospholipids confirmed the continued presence of $F$. succinogenes during the later stages of incubations on clover when this bacterium was not recovered from the roll tubes.

In the cultures containing both species, the analysis of phospholipids showed that $R$. flavefaciens rapidly outgrew $F$. succinogenes. Most earlier studies with mixed cultures of rumen cellulolytic micro-organisms have involved observations after 4 or (more commonly) $7 \mathrm{~d}$ incubation (Dehority \& Scott, 1967; Morris \& Van Gylswyk, 1980; Miron, 1991), by which time many of the faster growing species would have completed their degradation. The barley straw used here was the same as that used by Kolankaya et al. (1985), who reported that $R$. flavefaciens strain 007 degraded untreated barley straw more rapidly than did $F$. succinogenes strain $\mathrm{S} 85$; the maximum rate of straw solubilization during the first $48 \mathrm{~h}$ incubation with $R$. flavefaciens was approximately double that for $F$. succinogenes. Cell protein also accumulated more rapidly in supernatant liquids of the cultures containing $R$. flavefaciens, suggesting that this bacterium grew faster than $F$. succinogenes (Kolankaya et al., 1985), consistent with the results of the present study. After incubation for 8 d, Kolankaya et al. (1985) found that the strains of $R$. flavefaciens and $F$. succinogenes showed the same extent of straw solubilization (around $32 \%$ ). The present study, which was carried out with much greater quantities of plant material per incubation $(3.5 \mathrm{~g}$ versus $50 \mathrm{mg})$ could reasonably be expected to be more accurate, but the two strains of $F$. succinogenes used solubilized straw and clover both less rapidly and less extensively than the two strains of $R$. flavefaciens. The effects of the presence of two strains of the species were not investigated and possible interstrain interactions are an obvious topic for further investigation (Stewart et al., 1991), but it is considered that the use of multiple strains should more nearly approach the microbiological conditions in vivo.

Gram-positive cellulolytic cocci are normally recovered from the rumen in greater numbers than Gramnegative rods (Bryant \& Burkey, 1953; Kistner et al., 1962; Van Gylswyk, 1970; Mackie et al., 1978), in line with the predominance of $R$. flavefaciens in this study. The low numbers of $F$. succinogenes recovered from incubations performed with this species alone are puzzling. Analysis of the lipids associated with the residues did not suggest that $F$. succinogenes was resistant to detachment. Dehority (1968) reported that isolated hemicellulose can be degraded by cell-free extracts of rumen bacteria, but intact plant material would be expected to be more resistant to attack. Earlier observations by electron microscopy show that $F$. succinogenes proliferates to cover exposed plant cell wall surfaces during incubation with plant material (Cheng et al., 1983/84): it may be that these cells cannot be detached without losing viability.

In the rumen, the soluble products from the degradation of plant material that are not utilized by the major species of cellulolytic bacteria are presumably utilized by non-cellulolytic micro-organisms. Although $R$. flavefaciens was more active in solubilizing plant material than $F$. succinogenes, the latter, with its limited ability to utilize the products of hemicellulose hydrolysis (Morris \& Van Gylswyk, 1980) would be expected on the basis of this data to provide more arabinose and xylosecontaining substrates for interspecies cross-feeding. Xylose and glucose remained in the cultures after incubation mainly in the insoluble residues and as components of soluble degradation products, presumably di- or oligosaccharides, or glycosides. In contrast, 
significant amounts of arabinose tended to accumulate as the free sugar in incubations with either clover/ ryegrass or barley straw, and the accumulation of this free arabinose in the cultures containing the two bacterial species approximated to the sum of the amounts that accumulated in incubations that contained only one species. Significantly less soluble-bound arabinose accumulated in the cultures inoculated with both bacterial species than in cultures that received only one species. The characterization of the soluble components to which these monosaccharides were bound is an obvious topic of further interest. It should be remembered that the present study was carried out with plant material from which lipids had been extracted. In the rumen, the plant acyl lipids would be hydrolysed by lipolytic micro-organisms (Harfoot \& Hazlewood, 1988). In the present study the removal of the lipid component that cannot be hydrolysed by either of the bacterial species used would be expected to increase the apparent degradability of the substrates, but there is no evidence that the removal of lipids enhances plant cell wall degradation (Van Soest, 1981).

Several studies, mainly with Alfalfa (lucerne) have now demonstrated that the degradative activity of mixed cultures of primary rumen cellulolytic organisms including the ruminococci and $F$. succinogenes is usually less than that of the more active species growing in pure culture (Dehority \& Scott, 1967; Miron, 1991). A combined inoculum of $R$. albus and Clostridium longisporum was no more effective in the degradation of barley straw than $R$. albus alone (Varel et al., 1989). The present study specifically shows that the presence of 2 strains of $F$. succinogenes reduced the solubilization of clover/ryegrass by $R$. flavefaciens.

It is not clear why cellulolytic bacteria do not normally show complementary or synergistic activity in the solubilization of plant material. $R$. flavefaciens and $F$. succinogenes are thought to adhere to different plant tissue types (Latham et al., 1978), and these species do not show antagonistic or competitive interactions during their initial attachment to barley straw (Bhat et al., 1990). Both species require certain B-vitamins and branched-chain fatty acids (Hungate, 1966), however, in the present study there was no evidence of competition between the species for these nutrients. The bacteria may compete for colonization sites when they spread beyond the areas of initial attachment during the degradation. The bacteria with lower degradative activity (in this case, $F$. succinogenes) may thus reduce the access of the ruminococci to their substrate. This might explain why the numbers of viable bacteria recovered from the incubations tended to be greater in the absence of $F$. succinogenes, especially when clover/ryegrass served as substrate. Alternatively, the reduction by $R$. flavefaciens and $R$. albus of cellulolysis by some rumen fungi (Irvine \& Stewart, 1991; Bernalier et al., 1992) has been shown to involve the production by ruminococci of inhibitory compounds (Bernalier, 1991; Stewart et al., 1992) and it may be that similar interactions also occur between some species of rumen bacteria.

We thank Moira Thomas, Sylvia Duncan, A. J. Richardson and P. J. S. Dewey for their advice and help. We are also grateful to The Rotary International Freedom from Hunger Campaign, for their financial support for L.A.S., and the Scottish Office Agriculture and Fisheries Department who support A.S. and C.S.S.

\section{References}

Bernalier, A. (1991). Les champignons anaerobies du rumen: caracterisation et interactions avec les bacteries du rumen dans la cellulyse, in vitro. $\mathrm{PhD}$ thesis, Universite Blaise Pascal, Clermont Ferrand, France. No. d'ordre DU 281

Bernalier, A., Fonty, G., Bonnemoy, F. \& Gouet, F. (1992). Degradation and fermentation of cellulose by the rumen anaerobic fungi in axenic cultures or in association with cellulolytic bacteria. Current Microbiology 25, 143-148.

Bhat, S., Wallace, R. J. \& ORSKov, E. R. (1990). Adhesion of cellulolytic ruminal bacteria to barley straw. Applied and Environmental Microbiology 56, 2698-2703.

BoвbIE, R. J. \& White, D. C. (1980). Characterization of benthnic microbial community structure by high resolution gas chromatography of fatty acid methyl esters. Applied and Environmental Microbiology 39, 1212-1222.

Bryant, M. P. (1972). Commentary on the Hungate technique for the cultivation of strict anaerobes. American Journal of Clinical Nutrition 25, 1324-1328.

Bryant, M. P. \& Burkey, L. A. (1953). Numbers and some predominant groups of bacteria in the rumen of cows fed different rations. Journal of Dairy Science 36, 218-224.

Cheng, K.-J., Costerton, J. W., Dinsdale, D. \& Stewart, C. S. $(1983 / 84)$. Electron microscopy of bacteria involved in the digestion of plant cell walls. Animal Feed Science and Technology 10, 93-120.

Christie, W. W. (1982). Lipid Analysis: Isolation, Separation, Identification and Structural Analysis of Lipids. Oxford: Pergamon Press.

DeHORITY, B. A. (1968). Mechanisms of isolated hemicellulose and xylan degradation by celluloytic rumen bacteria. Applied Microbiology 16, 781-786.

DehoRity, B. A. \& SCOTT, H. W. (1967). Extent of cellulose and hemicellulose digestion in various forages by pure cultures of rumen bacteria. Journal of Dairy Science 50, 1136-1141.

DRUCKER, D. B. (1976). Gas-liquid chromatographic chemotaxonomy. Methods in Microbiology 9, 51-125.

Englyst, H. N. \& Cummings, J. H. (1984). Simplified method for the measurement of total non-starch polysaccharides by gas-liquid chromatography of constituent sugars as alditol acetates. Analyst 109, 937-942.

Feeding Stuffs (SAmpling and Analysis) Regulations (1982). Luff-Schoorl method. 10a, p. 29. London: HMSO.

Flint, H. J., McPherson, C. A. \& BissetT, J. (1989). Molecular cloning of genes from Ruminococcus flavefaciens encoding xylanase and $\beta(1-3,1-4)$ glucanase activities. Applied and Environmental Microbiology 55, 1230-1233.

Folch, J., LeEs, M. \& Stanley, G. H. S. (1957). A simple method for the isolation and purification of total lipids from animal tissues. Journal of Biological Chemistry 226, 497-509.

Harfoot, C. G. \& Hazlewood, G. P. (1988). Lipid metabolism in the rumen. In The Rumen Microbial Ecosystem, pp. 285-322. Edited by P. N. Hobson, London and New York: Elsevier Applied Science.

Hobson, P. N. (1969). Rumen bacteria. Methods in Microbiology 3B, $133-149$.

Hoebler, C., Barry, J. L., David, A. \& Delort-laval, Y. (1989). Rapid hydrolysis of plant cell wall polysaccharides and simplified 
quantitative determination of the neutral monosaccharides by gas-liquid chromatography. Journal of Agricultural and Food Chemistry 37, 360-367.

HUNGATE, R. E. (1966). The Rumen and its Microbes. New York: Academic Press.

HunGate, R. E. (1969). A roll tube method for cultivation of strict anaerobes. Methods in Microbiology 3B, 117-132.

IRVINE, H. L. \& STEWART, C. S. (1991). Interactions between anaerobic cellulolytic bacteria and fungi in the presence of Methanobrevibacter smithii. Letters in Applied Microbiology 12, 62-64.

Kistner, A., Gouws, L. \& Gilchrist, F. M. C. (1962). Bacteria of the ovine rumen. II. The functional groups fermenting carbohydrates and lactate on a diet of lucerne (Medicago sativa) hay. Journal of Agricultural Science 59, 85-91.

Kolankaya, N., Stewart, C. S., Duncan, S. H., Cheng, K.-J. \& COSTERTon, J. W. (1985). The effect of ammonia treatment on the solubilization of straw and the growth of cellulolytic rumen bacteria. Journal of Applied Bacteriology 58, 371-379.

Latham, M. J., Brooker, B. E., Pettipher, G. L. \& Harris, P. J. (1978). Adhesion of Bacteroides succinogenes in pure culture and in the presence of Ruminococcus flavefaciens to cell walls in leaves of perennial ryegrass (L. perenne). Applied and Environmental Microbiology 35, 1166-1173.

Mackie, R. I., Gilchrist, F. M. C., Robberts, A. M., Hannah, P. E. \& SChWARTz, H. M. (1978). Microbiological and chemical changes in the rumen during the stepwise adaptation of sheep to high concentrate diets. Journal of Agricultural Science 90, 241-254.

MCKinley, V. L. \& Vestal, J. R. (1984). Biokinetic analysis of adaptation and succession: microbial activity in composting sewage sludge. Applied and Environmental Microbiology 47, 933-941.

Minato, H. \& SuTo, T. (1981). Technique for fractionation of bacteria in rumen microbial ecosystem. IV. Attachment of rumen bacteria to cellulose powder and elution of bacteria attached to it. Journal of General and Applied Microbiology 27, 21-31.

Minato, H., Miyagawa, E. \& Suto, T. (1990). Techniques for analysis of rumen microbial ecosystems. In The Rumen Ecosystem, pp. 3-12. Edited by S. Hoshino, R. Onodera, H. Minato \& H. Itabashi. Tokyo: Japan Scientific Societies Press.

MiroN, J. (1991). The hydrolysis of lucerne cell-wall monosaccharide components by monocultures or pair combinations of defined ruminal bacteria. Journal of Applied Bacteriology 70, 245-252.

Miyagawa, E. (1982). Cellular fatty acid and fatty aldehyde com- position of rumen bacteria. Journal of General and Applied Microbiology 28, 389-408.

Mryagawa, E., Azuma, R. \& Suto, T. (1979). Cellular fatty acid composition in Gram-negative obligately anaerobic rods. Journal of General and Applied Microbiology 25, 41-51.

Morris, E. J. \& VAN GylswyK, N. O. (1980). Comparison of the action of rumen bacteria on cell walls of Eragrostis tef. Journal of Agricultural Science 95, 313-323.

StewarT, C. S. \& WALSH, J. H. (1972). Cellulolytic activity of pure and mixed cultures of fungi. Transactions of the British Mycological Society 58, 527-531.

Stewart, C. S., Flint, H. J. \& Nekrep, F. V. (1991). Degradation of plant cell wall polymers by the anaerobic rumen bacterium Ruminococcus flavefaciens. In Production and Utilization of Lignocellulosics, pp. 387-399. Edited by G. C. Galletti. London and New York: Elsevier Applied Science.

Stewart, C. S., Paniagua, C., Dinsdale, D., Cheng, K.-J. \& GARROW, S. H. (1981). Selective isolation and characteristics of Bacteroides succinogenes from the rumen of a cow. Applied and Environmental Microbiology 41, 504-510.

Stewart, C. S., Duncan, S. H., Richardson, A. J., Backwell, C. \& BEGBIE, R. (1992). The inhibition of fungal cellulolysis by cell-free preparations from ruminococci. FEMS Microbiology Letters 97, 83-88.

VAN GYLSWYK, N. O. (1970). The effect of supplementing a low protein hay on the cellulolytic bacteria in the rumen of sheep and on the digestibility of cellulose and hemicellulose. Journal of Agricultural Science 74, 169-180.

VAN SOEST, P. J. (1981). Limiting factors in plant residues of low biodegradability. Agriculture and Environment 6, 135-143.

VARel, V. H., Richardson, A. J. \& Stewart, C. S. (1989). Degradation of barley straw, ryegrass, and alfalfa cell walls by Clostridium longisporum and Ruminococcus albus. Applied and Environmental Microbiology 55, 3080-3084.

Verkley, A. J., Ververgaert, P. H. J. T., Prins, R. A. \& Van Golde, L. M. G. (1975). Lipid-phase transitions of the strictly anaerobic bacteria Veillonella parvula and Anaerovibrio lipolytica. Journal of Bacteriology 124, 1522-1528.

VESTAL, J. R. \& WhITE, D. C. (1989). Lipid analysis in microbial ecology. Bioscience 39, 535-541.

Watanabe, T., OKuda, S.-I. \& Takahashi, H. (1982). Physiological importance of even numbered fatty acids and aldehydes in plasmalogen phospholipids of Selenomonas ruminantium. Journal of General and Applied Microbiology 28, 23-33. 